\section{Challenges in Chronic Genetic Disorders: Lessons From the COVID-19 Pandemic}

To examine the impact of the COVID-19 pandemic, we interviewed 26 patients with lysosomal storage disorders receiving enzyme replacement therapy. $20(77 \%)$ had significant interruption in their treatment, with an average of 8 (range 2-28) missed doses. Alternate methods of delivering uninterrupted care including home therapy were used. Vulnerable patients with chronic genetic disorders require organization for their multidisciplinary needs of care.

Keywords: Enzyme replacement therapy, Lysosomal storage disorders.

The unprecedented COVID-19 epidemic had healthcare at its epicenter. SARS-CoV-2 infection has been reported to have a worse outcome in patients with co-morbidities [1]. Patients with genetic disorders having multisystem involvement comprise a vulnerable high risk population [2]. Over the years, with improved awareness and advocacy, some patients with lysosomal storage disorders (LSD) receive intravenous infusions of enzyme replacement therapy (ERT) [3]. This management and associated multidisciplinary care are ordinarily challenging with significant impact on the family [4]. With the strict nationwide lockdown, we hypothesized that the pandemic brought new challenges for these patients. To evaluate the multifaceted impact on LSD patients receiving ERT, we carried out this telephonic survey using a dataset questionnaire.

Twenty six patients (20 males) receive ERT from our center. Their median (range) age was 18 (3-53) years. The average (range) duration of ERT was 5.6 years ( 2 months to 12 years). Eleven patients with Gaucher disease (42\%) were receiving imiglucerase or velaglucerase, five each (19\%) with MPS I and Fabry disease receiving laronidase and agalsidase beta, respectively, three (12\%) with MPS II receiving idursulfase and two (8\%) with Pompe disease receiving alglucosidase $\alpha$.

Twenty patients (77\%) had an interruption of two or more doses of ERT, while one patient missed a single dose. An average of 8 doses (range 2 to 28) were missed of a total of 28 doses for weekly infusion and 14 doses for two weekly infusion that should have been received during the study period. Two patients experienced a delay of one week in their regular schedule. One Fabry disease patient had to delay the start of his ERT. Two patients $(7.7 \%)$ did not experience any interruption in their schedule and both were below 18 years of age under parental supervision. Of 20 patients who missed multiple doses, thirteen $(65 \%)$ could not collect the medicine from the tertiary centre due to lack of transportation, while for two (10\%) the medicine import was delayed. Four patients did not get the infusion as the local hospital had closed and one patient avoided going to the hospital for fear of infection (Fig. 1). Two patients each of Fabry and Gaucher disease switched to home based ERT and two more patients specifically mentioned wanting to do the same.

Most patients did not report any acute worsening or new symptoms. One Fabry disease patient experienced increased pain and a rising serum creatinine level and one patient with Gaucher disease reported increased weakness and bone pain. Twelve patients or their parents (46\%) reported anxiety and fear of the ongoing pandemic. Of these, $66 \%$ complained of constant worry, that exacerbated during hospital visits for ERT, while others combated their fear by strict adherence to social distancing and use of masks.

Majority of our patients missed multiple doses of ERT. The medicines are imported on a named patient basis and maintained at the tertiary care centre. These are collected at predefined intervals and infused at a local health care facility. The patients are followed up at 3-6 monthly intervals at the tertiary-facility. Most patients were unable to reach the tertiary facility due to lack of transportation and the centers being far away.

In contrast to Western countries, there is no system for home-based ERT in India. The pandemic created a need wherein patients and families identified local means to continue infusions. Studies from countries in Europe showed significant interruption in hospital-based ERT in up to 49\% LSD patients in Italy and $25 \%$ of Gaucher disease patients in Spain, while patients on home therapy received regular treatment $[5,6]$. In our study, $15 \%$ patients switched to home-based therapy while an additional $7.7 \%$ wished to do so. The constant fear faced by patients' families for the affected member contracting COVID19 infection was increased during hospital visits due to increased risk of exposure.

The long term impact of ERT interruption needs follow up. Previous experience with ERT interruption is limited and suggests that after restarting ERT, clinical deterioration may not be completely reversible [7-9]. While ERT is largely considered

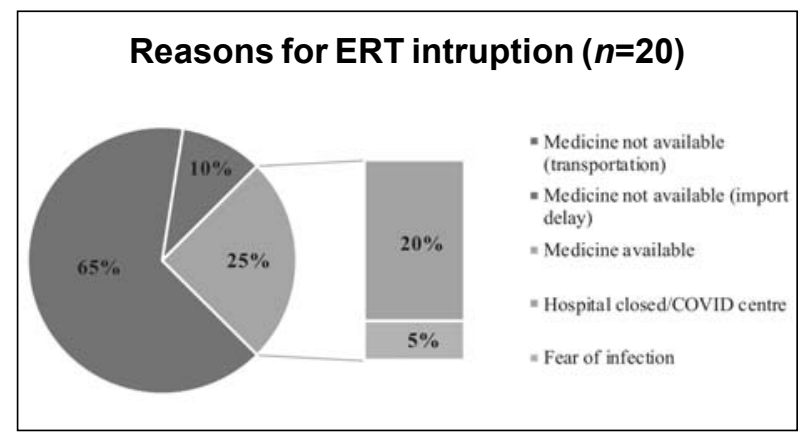

Fig. 1 Patients with significant interruption in enzyme replacement therapy and causes for the same. 
safe, adverse events are reported. Though the circumstances promoted the shift to home based therapy, we wonder if the preparedness is adequate.

The bi-annual surveillance with clinical examination and blood and radiological investigations were missed by all twenty-six patients. As the therapy is relatively new, expensive, with stringent storage requirements, management is through tertiary care centres and primary care physicians are mostly not involved in patient treatment and follow-up. This lack of experience and awareness at the peripheral health centres impacted local management for these patients.

This study highlights pertinent aspects of interruption to care, anxiety and concerns of patients and their families with chronic genetic disorders, and the limitations of a tertiary care centric management for chronic disorders. The emerging role of telemedicine as an important tool for follow up and care of patients with chronic disorders is important. As we strive to increase access to ERT, we recognize the need to equip peripheral centres for care of patients with genetic disorders along with consultation with tertiary centres.

As the pandemic continues, we still grapple without guidelines to manage chronic genetic disorders. There is an urgent need to draw the attention of medical authorities to facilitate multi-specialty care for these patients to prepare for similar unforeseen situations.

Ethical clearance: No. IEC SGRH; EC/12/20/1784, dated January 12, 2021.

Contributors: SP, SB: conception of work, acquisition, analysis and interpretation of data, drafting and revising the work; SBM: drafting and revising the work; ICV: drafting the work and revising it critically for important intellectual content; RDP: conception and design of work, Interpretation of data, drafting and revising the work. All authors approved the final version of manuscript.

Funding: None for this study; Competing interests: None stated.
Swasti Pal, Sameer Bhatia, Sunita Bijarnia-Mahay, Ishwar C Verma and Ratna Dua PURI* Institute of Medical Genetics and Genomics, Sir Ganga Ram Hospital, New Delhi, India.

*ratnadpuri@yahoo.com

\section{REFERENCES}

1. Yang J, Zheng Y, Gou X, et al. Prevalence of comorbidities and its effects in patients infected with SARS-CoV-2: A systematic review and meta-analysis. Int J Infect Dis. 2020;94:91-95.

2. Elmonem MA, Belanger-Quintana A, Bordugo A, et al. The impact of COVID-19 pandemic on the diagnosis and management of inborn errors of metabolism: A global perspective. Mol Genet Metab. 2020:S1096-7192(20)30195-5.

3. Muranjan M, Karande S. Enzyme replacement therapy in India: Lessons and insights. J Postgrad Med. 2018;64:195-99.

4. Hamad L, Kreidieh K, Nakouzi G, Lyon E, Yazbek S. Recommended measures for the efficient care of patients with genetic disorders during the COVID-19 pandemic in low and middle income countries. Am J Med Genet A. 2020 Oct 16. doi:10.1002/ ajmg.a.61879 [Epub ahead of print].

5. Sechi A, Macor D, Valent S, et al. Impact of COVID-19 related healthcare crisis on treatments for patients with lysosomal storage disorders, the first Italian experience. Mol Genet Metab. 2020;130:170-71.

6. Andrade-Campos M, Escuder-Azuara B, de Frutos LL, SerranoGonzalo I, Giraldo P. Direct and indirect effects of the SARS-CoV2 pandemic on Gaucher Disease patients in Spain: Time to reconsider home-based therapies? Blood Cells Mol Dis. 2020;85:102478.

7. Giraldo P, Irún P, Alfonso P, et al. Evaluation of Spanish Gaucher disease patients after a 6-month imiglucerase shortage. Blood Cells Mol Dis. 2011;46:115-8.

8. Solano M, Fainboim A, Politei J, et al. Enzyme replacement therapy interruption in patients with Mucopolysaccharidoses: Recommendations for distinct scenarios in Latin America. Mol Genet Metab Rep. 2020;23:100572.

9. Politei J. Fabry disease during the COVID-19 pandemic. Why and how treatment should be continued. Mol Genet Metab. 2020;130:227-29.

\section{Appropriateness of Lower Waist Circumference Cutoffs for Predicting Derangement in Metabolic Parameters Among Asian Children and Adolescents: A Pilot Study}

Waist circumference (WC) >90th percentile cut-off effectively screens children for metabolic syndrome, as some specific metabolic derangements (high fasting serum levels of insulin and triglycerides) may be better associated with lower (70th percentile) waist circumference cut off. We evaluated a subset of children and adolescents found obese or overweight following the anthropometric screening in a school-based survey. Metabolic parameters (fasting insulin levels, fasting blood sugar and fasting lipid profile and blood pressure) were compared among 3 groups of obese or overweight children divided on the basis of WC percentiles (>90th, 70th-90th and $<70$ th). 78 children (aged 11-18 years, 45 boys) were evaluated. The proportion of participants with high triglycerides and fasting insulin among those with WC $<70$ th $(28.6 \%, 19 \%)$ was significantly lower than that in the group with WC $>90$ th $(76.9 \%, 53.8 \%)$ as well as in group with WC 70th-90th percentile (38.7\%, 41.9\%).

Keywords: Anthropometry, Blood sugar, Central obesity, Overweight, Triglycerides.

Obese children from south Asian region are relatively more prone to develop components of metabolic syndrome [1]. Waist circumference is a widely accepted and practical tool for community screening of obesity [2]. However, certain concerns have been raised over the cutoff being used while utilizing waist circumference as a predictor of metabolic syndrome [3,4]. We explored the suitability of lower waist circumference cutoff 